\title{
Efficient Transmission of Watermarked Images with SC-FDMA
}

\author{
Fatma. E .Fadl Allah \\ Dept. of Electronics and \\ Communication Engineering, \\ Faculty of Engineeing, \\ Zagazig University, \\ Zagazig, Egypt \\ fatmaelsaid19@yahoo.com
}

\author{
Naglaa. F. Soliman \\ Dept. of Electronics and \\ Communication Engineering, \\ Faculty of Engineeing, \\ Zagazig University, \\ Zagazig, Egypt \\ nagla_soliman@yahoo.com
}

\author{
M. I. Abdalla \\ Dept. of Electronics and \\ Communication Engineering, \\ Faculty of Engineeing, \\ Zagazig University, \\ Zagazig, Egypt \\ mabdalla2010@gmail.com
}

\author{
Fathi E. Abd El-Samie \\ Dept. of Communications, \\ Faculty of Electronic Engineeing, \\ Menofia University, \\ Menouf, Egypt \\ fathi_syed@yahoo.com
}

\begin{abstract}
This paper studies the transmission of watermarked images on wireless SC-FDMA systems. Different watermarking schemes are provided in this frame work for security and authentication purposes.SC-FDMA is accomplished for broadband wireless communications, for its advantages of low PAPR and low sensitivity to carrier frequency offsets. The SC-FDMA system is used for communication as it is the uplink standard in coefficient mobile communication system. In this paper, two types of equalizers(MMSE and ZF) is used to mitigate the effect of channel fading and noise at different subcarriers mapping schemes(localized and distributed).Watermark extraction is performed at the receiver and an evaluation process for the quality of extracted watermarks is performed. Simulation experiments were established in MATLAB program R2014a version. Simulation results approved that the interleaved system provides better performance than that of the localized systems. In addition the system performance has better enhancement when using the $\mathrm{ZF}$ equalizer than the MMSE equalizer. The quality of the received of extracted watermarks is investigated with different metrics like PSNR, correlation coefficient $(\mathrm{Cr})$, SSIM and the histogram . Simulation results also demonstrate an efficient of image clarity at extraction process.
\end{abstract}

Keywords - SC-FDMA, Image Water marking, DCT, DWT.

\section{Introduction}

By the quick growth of computer network in the latest years we can process digital contents and access to various digital media easily. Therefore media security became a highly important issue [1].Image watermarking is the process of embedding some useful information inside images for the purpose of security and authentication of the source. Different algorithms of digital image watermarking have been developed. In this paper, two different techniques of digital image watermarking have been used, DCT and DWT.

Single carrier frequency division multiple access (SCFDMA) is a superior technology for uplink broadband wireless communications, as it does not have the problem of the large Peak-to-Average Power Ratio(PAPR) as opposed to orthogonal frequency division multiple Access (OFDMA)[2].

In image watermarking transmission system, It is highly important to study the images performance with watermarks embedded and extracted through SC-FDMA channel transmission. This paper has studied the performances of two different watermarking algorithms (DCT and DWT) through SC-FDMA channel[1].

The algorithm performance is measured by these important requirements of digital watermarking PSNR, $\mathrm{Cr}$ and SSIM .The PSNR in $\mathrm{dB}$ to measure the watermarked image imperceptibility, correlation of original mark image and recovering mark image to measure robustness of the mark image and the SSIM [3,11]. For this study a $512 \times 512$ gray scale "Lena" image is used as cover image, and a $50 \times 20$ copy right image is used as mark image .

\section{Image Watermarking over The SC-FDMA System}

Figure 1 shows the image watermarking over the SCFDMA system architecture. The watermark embedding block is used for embedding the watermark into the cover image with the used algorithm either in DCT or DWT digital image watermarking technique. The image formatting converts the image to a binary form that has become suitable to be inserted, processed and transmitted over the SC-FDMA system. The SC-FDMA transmitter starts with an encoder then a modulation of the input signal using binary Quadrature amplitude modulation (QAM). The modulated symbols are grouped into blocks, each block containing $N$ symbols and the FFT is performed. The signal after the FFT is expressed in Eq. (1) [7] :

$$
X(k)=\sum_{n=0}^{N-1} X(n) e^{\frac{-j 2 \pi}{N} n k}
$$

where $N$ is the input block size, $\{x(n): \mathrm{n}=0, \ldots, N-1\}$ 
represents the modulated symbols.

The outputs after the FFT are mapped to M orthogonal subcarriers followed by the M-points IFFT. The resulting signal after the IFFT can be given as in Eq. (2)[7]:

$$
\bar{X}(m)=\frac{1}{M} \sum_{\mathbb{l}=0}^{M-1} \bar{X}(\mathbb{l})_{e} \frac{j 2 \pi}{M} m \mathbb{l}
$$

where : $\{\bar{X}(l): l=0, \ldots, M-l\}$ is the frequency-domain samples after the subcarriers mapping scheme.

Cyclic Prefix (CP) which is a group of symbols is also inserted to give a guard time that is very important to forbid Inter-Block Interference (IBI) caused by multipath propagation. $\mathrm{CP}$ is a copy of the end part of the block. It is inserted at the start of each block. The transmitted data crossed through the channel $[4,5]$.

At the receiver, the $\mathrm{CP}$ is removed, and after that the received signal is converted to the frequency domain by (FFT). The samples are crossed through the Frequency Domain Equalizer (FDE) then the de-mapping operation isolates the frequency-domain samples of each source signal. The Inverse Fast Fourier Transform (IFFT) at the receiver transforms equalized symbols back to the time domain. The demodulation process retrieves the original data, passed through the decoder. The image reconstruction converts the binary form to an image for recovering the original image. The watermark is extracted from the watermarked image by Watermark extraction [4,5].

\section{Subcarriers Mapping Methods}

The localized and distributed mapping methods are two schemes for mapping the subcarriers between users. The first one is ordinarily called localized scheme LFDMA, while the other one is ordinarily referred to as distributed FDMA transmission scheme. With the LFDMA transmission technique, each user's data is transported with consecutive subcarriers. In the distributed transmission scheme, the user's data is transported with distributed subcarriers. Because of The information symbol spreading through the entire signal band, the distributed FDMA technique is more strong to frequency-selective fading. Therefore, distributed scheme can realize more frequency diversity. For the transmission of localized through a frequency-selective fading channel, both the diversity of the multiuser and the frequency-selective diversity can be realized if each user has got subcarriers with appropriate transmission characteristics. The distributed FDMA technique that has equidistance among occupied subcarriers for the whole of the band is referred to as the interleaved FDMA scheme (IFDMA) [6,7].

Figure 2 illustrates An example for the two Methods for3 users, 12 subcarriers, and 4 subcarriers assigned per user. For this paper, the localized SC-FDMA system is referred to as LFDMA, the interleaved SC-FDMA system is called IFDMA [6,7].

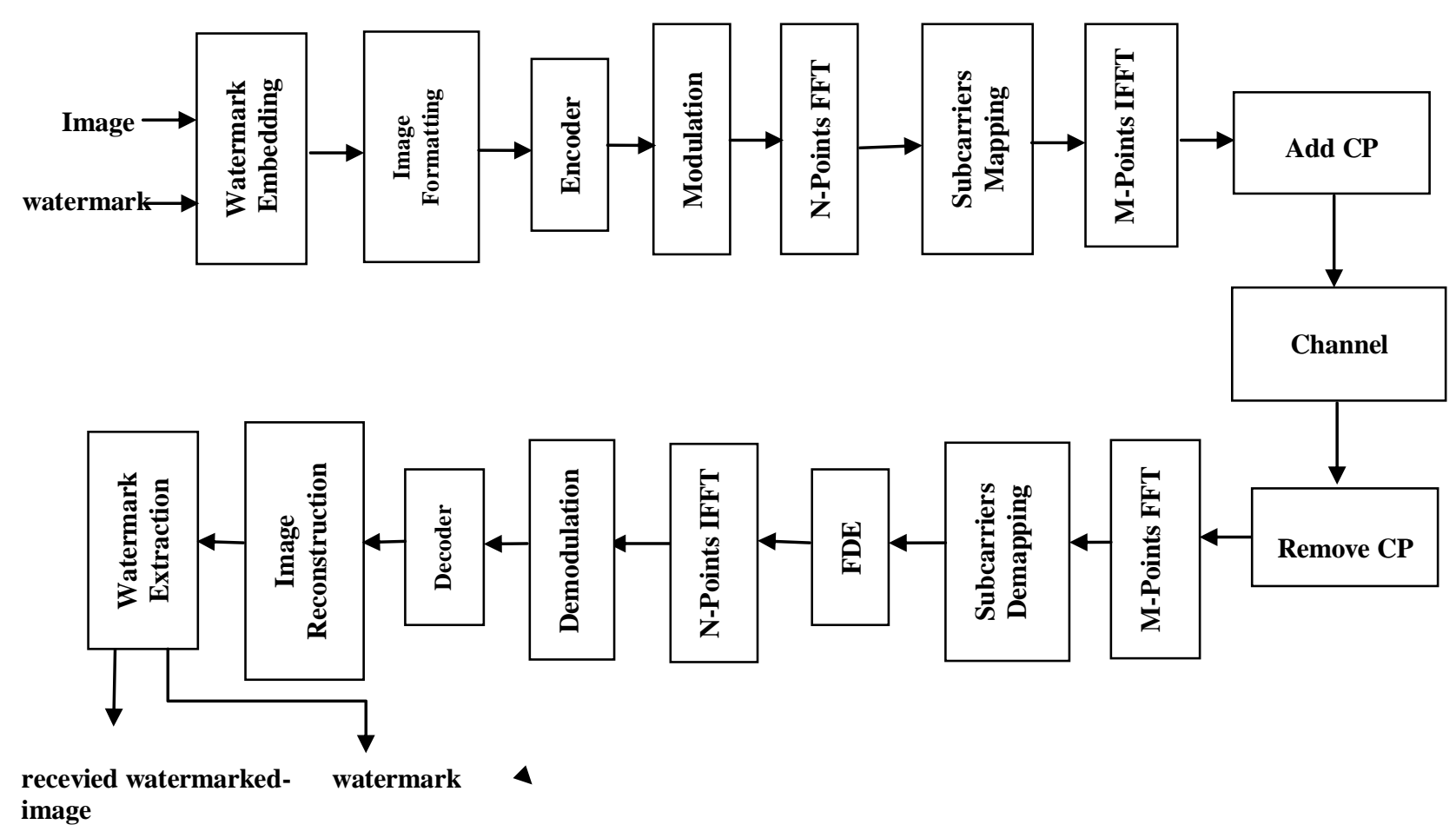

Fig. 1 Image Watermarking over the SC-FDMA System 


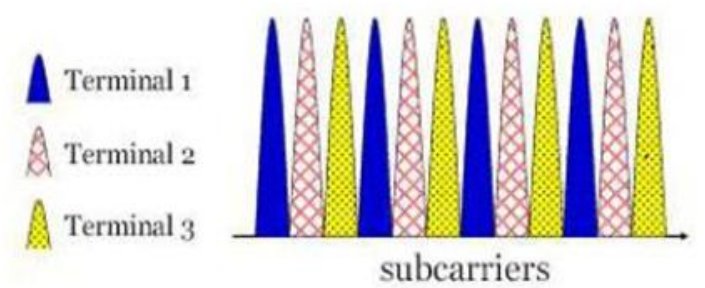

Distributed Mode

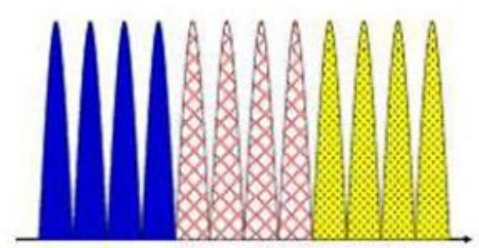

subcarriers

Localized Mode

Fig. 2 Subcarriers mapping schemes for multiple users (3 users, 12 subcarriers, and 4 subcarriers assigned per user) [6].

\section{Image Watermarking Algorithms}

Watermarking is a copyright protection technique that permits tracking back-illegally created copies of the protected multimedia contents. Compared with other copyright protection techniques, the watermarking main advantage is that the watermark is embedded permanently in the content of the visual data, but at the cost of a slight loss in fidelity. Also, The watermarking can reduce the required bandwidth for data transmission.

Digital watermarking has different schemes for protecting the digital content. The digital image watermarking schemes are based on two domains, one is the spatial domain scheme and the second is transform domain scheme. The spatial domain scheme processes pixels directly. It embeds the watermark by processing the pixels value. LSB is the Most popular used spatial domain scheme. Transform domain scheme embed the watermark by processing the transform domain coefficients. Most popular used transform domain scheme is DCT, DWT and DFT. This paper is depends on DCT scheme and DWT scheme [8]

\subsection{Discrete Cosine Transform (DCT)}

Discrete Cosine Transform is a highly favorable transform domain watermarking technique. In which the image is split into various frequency band, low FL, medium FM and high FH band. It permits selecting the band for embedding watermark into the image. Figure 3 shows the three frequency region of DCT of 8x8 block. Low frequency band FL is located in upper left corner, in case modification performed here, human eyes can discover the watermark. High frequency band FH appears at lower and right edges, if modification performed here, it may cause of local distortion along with edges. Medium frequency band FM is considered perfect region for modification as it cannot influence the quality of the image. Thus, FM is the perfect band for embedding watermark [9].

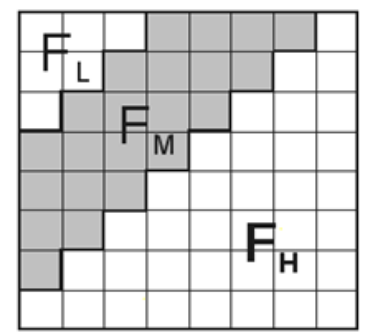

Fig.3 Definition of DCT Regions for 8X8 block [9].

\section{The DCT watermark embedding algorithm can be illustrated as follows:}

1. Read the cover image to be watermarked

2. Segment the image into non-overlapping blocks of $8 \mathrm{x} 8$

3. Apply forward DCT to each of these blocks as in Eq. (3)[10]

$$
\begin{aligned}
& B_{p q}= \\
& \alpha_{p} \alpha_{q} \sum_{m=0}^{M-1} \sum_{n=0}^{N-1} A_{m n} \cos \frac{\pi(2 m+1) p}{2 M} \cos \frac{\pi(2 n+1) q}{2 N}{ }^{0} 0 \leq P \leq M-1 \\
& 0 \leq q \leq N-1
\end{aligned}
$$

where,

$$
\alpha_{p}= \begin{cases}\frac{1}{\sqrt{M}}, & P=0 \\ \sqrt{\frac{2}{M}}, & 1 \leq P \leq M-1\end{cases}
$$

and

$$
\alpha_{q}= \begin{cases}\frac{1}{\sqrt{N}}, & q=0 \\ \sqrt{\frac{2}{N}}, & 1 \leq q \leq N-1\end{cases}
$$

$\mathrm{M}$ and $\mathrm{N}$ are the row and column size of image $\mathrm{A}$, respectively.

4. Read and reshape the watermark image

5. Check that the watermark image isn't too large for cover

6. Obtaining the watermarked image DCT components by adding the watermark and the image DCT coefficients in the mid band frequency

7. Get the watermarked image by applying the inverse DCT transform for all sub-blocks.

The watermarked image is transmitted through the SC FDMA system on AWGN channel. Finally, at the receiver the watermark image has been extracted.

\section{The DCT watermark extraction algorithm is explained in the following steps :}

1. Segment the watermarked image into nonoverlapping blocks of $8 \times 8$ 
2. Apply forward DCT to each of these blocks.

3. Extract the DCT components of the image from the watermarked image DCT coefficients.

4. Get watermark image.

5. Calculate for PSNR, Cr, and SSIM.

\subsection{Discrete Wavelet Transform (DWT):}

The single DWT divides two dimensional images into four sub-bands: one sub-band contains the original image low frequency LL, the top right includes horizontal details of the image $\mathrm{HL}$, the one bottom left Includes vertical details of the original image $\mathrm{LH}$, the bottom right Includes high frequency of the original image $\mathrm{HH}$. The low frequency LL is split again into two more parts of high and low frequencies, this process is repeated to have multilevel wavelet components like 2-Level, 3-Level etc. until the signal has been completely decomposed. Fig. 4is a TwoLevel Discrete Wavelet Transform. The coefficients of the low frequency are more robust to embed watermark as it includes more information of the original image $[8,9]$.

\begin{tabular}{|c|c|c|}
\hline LL2 & HL2 & \multirow{2}{*}{ HL1 } \\
LH2 & HH2 & \\
LH1 & \multirow{2}{*}{ HH1 } \\
\hline
\end{tabular}

Fig.4 2-Level Discrete Wavelet Transform [9].

\section{The DWT Watermark embedding algorithm :}

1. Read the cover image to be watermarked.

2. Read and reshape the watermark image.

3. Read in key for PN generator.

4. Apply 2nd level DWT to the cover image.

5. Embed the components of watermark in to the frequency subcomponents

6. Apply IDWT.

7. Get watermarked image.

After transmitting the watermarked image through the SC FDMA system, watermark has been extracted.

\section{The DWT Watermark extraction algorithm:}

1. Read Watermarked image.

2. Apply DWT.

3. Extract the components of watermark from frequency subcomponents .

4. Get watermark image.

5. Calculate for PSNR, Cr, and SSIM.

\section{Quality Measurement Parameters}

PSNR, Correlation (Cr) and SSIM are very good parameters used for investigating the image quality. These parameters can be briefly defined as follow:

\subsection{Peak signal to noise ratio (PSNR)}

The PSNR is used for measuring the quality of extracted image Compared to the original images after the receiving process. The ratio between the signal maximum power and the noise power is called PSNR. The optimum case for the PSNR value is to be as high as possible. It can be mathematically calculated in Eq. (4) [11].

$$
\operatorname{PSNR}(d B)=10 \log \left(\frac{P^{2}{ }_{\text {Max }}}{M S E}\right)=20 \log \left(\frac{P_{\text {Max }}}{\sqrt{M S E}}\right)
$$

The $\mathrm{P}_{\mathrm{Max}}$ is the higher pixel value, for maximum value of the image pixel, $\mathrm{P}_{\mathrm{Max}}=2 \mathrm{~b}-1, \mathrm{~b}$ is the bits number per sample. The MSE (mean square error) is a tool for measuring the average of squares of errors, here used for measuring the difference between the original cover image and the received watermarked image. It is calculated mathematically in Eq. (5) [11].

$$
M S E=\frac{1}{M N} \sum_{i=0}^{M-1} \sum_{j=0}^{N-1}\left[f\left(i_{i} j\right)-f^{n}\left(i_{i} j\right)\right]^{2}
$$

where $f(i, j)$ is the original cover image version and $\mathrm{f}^{t}\left(i_{s} j\right)$ is the received watermarked image.

\subsection{Correlation coefficients $(\mathrm{Cr})$}

$\mathrm{Cr}$ is a tool measures the convergence degree between the original mark image and the extracted mark image at the receiver side, so it gives a direct measure of proposed algorithm performance. The optimum case produces images with correlation ratio so close to unity. Correlation coefficients of two images A and B can be found from Eq. (6) $[3,11]$.

Corr $=\frac{\Sigma_{m} \Sigma_{n}\left(A_{m n}-\bar{A}\right)\left(B_{m n}-\bar{B}\right)}{\sqrt{\left(\left(\sum_{m} \Sigma_{n}\left(A_{m n}-\bar{A}\right)^{2}\right)\left(\sum_{m} \Sigma_{n}\left(B_{m n}-\bar{B}\right)^{2}\right)\right.}}$

where $\bar{A}=\operatorname{mean} 2(A)$, and $\bar{B}=\operatorname{mean} 2(B)$.

\subsection{Structural Similarity Index Measurement (SSIM)}

SSIM is an image fidelity measure that has proved highly effective in measuring signals fidelity. The SSIM approach was originally motivated by the observation that the human visual system is adapted for extracting structural information from images. so, it is highly important keeping the structural signal for image quality measurement $[12,13]$. SSIM is used to measure similarity between original image and watermarked image or watermark and recovered watermark based on statistical parameters such as mean and variance of images. Its value exit between the decimal values 1 and - 1 . If SSIM is equal to 1 , it means the images are identical sets. It is calculated as in Eq. (7):

$\operatorname{SSIM}(x, y)=\frac{\left(2 \mu_{x} \mu_{y}+C 1\right)\left(2 \sigma_{x y}+C 2\right)}{\left(\mu_{x}^{2}+\mu_{y}^{2}+C 1\right)\left(\sigma_{x}^{2}+\sigma_{y}^{2}+C 2\right)}$ 
where, $\mu_{x}$ is average of $\mathrm{x}, \mu_{y}$ is average of $\mathrm{y}, \sigma_{x}^{2}$ is the variance of $\mathrm{x}, \sigma_{y}^{2}$ is the variance of $\mathrm{y}, \sigma_{x y}$ is the covariance of $\mathrm{x} \& \mathrm{y}, \mathrm{C} 1$ and $\mathrm{C} 2$ are the two variable to stabilize the division with weak denominator[9].

\section{Simulation Results}

In this section, the watermarked images performance after transmission on a wireless environment by using SCFDMA technique have been studied for different types of equalizers, MMSE and ZF equalizer[1].Simulation parameters are tabulated in Table 1.
Table1: Simulation parameters

\begin{tabular}{|l|l|}
\hline Parameter & Value \\
\hline FFT size & 512 symbols \\
\hline Input block size & 128 symbols \\
\hline Cyclic prefix size & 20 samples \\
\hline Cover Image size & $512 \times 512$ \\
\hline Mark image size & $50 \times 20$ \\
\hline Modulation type & QAM \\
\hline Subcarriers mapping & Interleaved and localized \\
\hline Noise environment & AWGN \\
\hline Equalizer type & MMSE and ZF \\
\hline
\end{tabular}

Table 2: PSNR values of the received DCT and DWT watermarked image over the SC-FDMA system

\begin{tabular}{|c|c|c|c|c|c|c|c|c|}
\hline \multirow{3}{*}{ 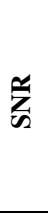 } & \multicolumn{4}{|c|}{ DCT } & \multicolumn{4}{|c|}{ DWT } \\
\hline & \multicolumn{2}{|c|}{ MMSE } & \multicolumn{2}{|c|}{$Z F$} & \multicolumn{2}{|c|}{ MMSE } & \multicolumn{2}{|c|}{$Z F$} \\
\hline & $\begin{array}{l}\mathbf{3} \\
\mathbf{3}\end{array}$ & $\underset{3}{\mathbb{S}}$ & $\begin{array}{l}\mathbf{3} \\
\mathbf{s} \\
\mathbf{s}\end{array}$ & 妾 & $\begin{array}{l}\mathbb{S} \\
\mathbf{S}\end{array}$ & $\underset{3}{3}$ & $\begin{array}{l}\mathbb{Z} \\
\mathbf{Z}\end{array}$ & $\underset{S}{S}$ \\
\hline 0 & 9.850 & 9.735 & 8.801 & 7.939 & 9.770 & 9.542 & 8.814 & 7.939 \\
\hline 2 & 10.242 & 10.213 & 9.568 & 8.543 & 10.189 & 10.150 & 9.586 & 8.545 \\
\hline 4 & 10.378 & 10.220 & 10.381 & 9.244 & 10.352 & 10.293 & 10.421 & 9.251 \\
\hline 6 & 10.483 & 10.141 & 11.145 & 10.078 & 10.552 & 10.377 & 11.207 & 10.087 \\
\hline 8 & 11.127 & 10.834 & 12.062 & 10.969 & 11.205 & 10.962 & 12.141 & 10.990 \\
\hline 10 & 12.043 & 11.880 & 13.664 & 11.876 & 12.151 & 11.922 & 13.716 & 11.918 \\
\hline 12 & 13.444 & 13.001 & 15.131 & 12.906 & 13.571 & 13.014 & 15.139 & 12.956 \\
\hline 14 & 15.485 & 14.540 & 17.217 & 14.047 & 15.634 & 14.491 & 17.110 & 14.083 \\
\hline 16 & 18.510 & 16.341 & 20.398 & 15.592 & 18.511 & 16.241 & 19.988 & 15.576 \\
\hline 18 & 23.048 & 18.803 & 29.003 & 17.913 & 22.467 & 18.522 & 26.128 & 17.766 \\
\hline 20 & 30.149 & 22.105 & 34.020 & 21.170 & 26.923 & 21.446 & 27.939 & 20.678 \\
\hline
\end{tabular}

Table $3 \mathrm{Cr}$ values of the extracted watermark based DCT and DWT watermarking technique.

\begin{tabular}{|c|c|c|c|c|c|c|c|c|}
\hline \multirow{3}{*}{ 点 } & \multicolumn{4}{|c|}{ DCT } & \multicolumn{4}{|c|}{ DWT } \\
\hline & \multicolumn{2}{|c|}{ MMSE } & \multicolumn{2}{|c|}{$Z F$} & \multicolumn{2}{|c|}{ MMSE } & \multicolumn{2}{|c|}{$Z F$} \\
\hline & $\begin{array}{l}\mathbb{s} \\
\mathbf{s}\end{array}$ & $\underset{3}{\mathbb{s}}$ & $\begin{array}{l}\mathbb{3} \\
\mathbf{s}\end{array}$ & $\underset{3}{\mathbb{S}}$ & S & $\underset{\mathbf{S}}{\mathbb{S}}$ & S & $\underset{⿱ 乛 龰}{\mathbf{S}}$ \\
\hline 0 & 0.029 & 0.167 & 0.144 & 0.270 & 0.400 & 0.355 & 0.557 & 0.546 \\
\hline 2 & 0.117 & 0.176 & 0.163 & 0.334 & 0.424 & 0.387 & 0.621 & 0.574 \\
\hline 4 & 0.144 & 0.144 & 0.274 & 0.307 & 0.453 & 0.425 & 0.693 & 0.574 \\
\hline 6 & 0.196 & 0.247 & 0.329 & 0.305 & 0.493 & 0.456 & 0.759 & 0.652 \\
\hline 8 & 0.272 & 0.267 & 0.405 & 0.384 & 0.511 & 0.498 & 0.805 & 0.707 \\
\hline 10 & 0.290 & 0.338 & 0.507 & 0.496 & 0.571 & 0.549 & 0.859 & 0.741 \\
\hline 12 & 0.403 & 0.450 & 0.709 & 0.634 & 0.633 & 0.602 & 0.886 & 0.809 \\
\hline 14 & 0.534 & 0.599 & 0.735 & 0.693 & 0.733 & 0.676 & 0.902 & 0.852 \\
\hline 16 & 0.728 & 0.739 & 0.876 & 0.874 & 0.874 & 0.765 & 0.969 & 0.890 \\
\hline 18 & 0.893 & 0.892 & 1 & 0.941 & 0.969 & 0.874 & 1 & 0.941 \\
\hline 20 & 0.989 & 0.974 & 1 & 1 & 1 & 0.950 & 1 & 0.979 \\
\hline
\end{tabular}


Table 4 SSIM values of the received watermarked image based DCT and DWT over the SC-FDMA system.

\begin{tabular}{|c|c|c|c|c|c|c|c|c|}
\hline \multirow{3}{*}{$\underset{m}{\mathbf{Z}}$} & \multicolumn{4}{|c|}{ DCT } & \multicolumn{4}{|c|}{ DWT } \\
\hline & \multicolumn{2}{|c|}{ MMSE } & \multicolumn{2}{|c|}{$Z F$} & \multicolumn{2}{|c|}{ MMSE } & \multicolumn{2}{|c|}{$Z F$} \\
\hline & S & 羟 & S & S & S & 就 & S & 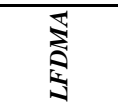 \\
\hline 0 & 0.1421 & 0.1480 & 0.0729 & 0.0539 & 0.1378 & 0.1470 & 0.0720 & 0.0530 \\
\hline 2 & 0.1151 & 0.0942 & 0.0952 & 0.0718 & 0.1166 & 0.0989 & 0.0936 & 0.0703 \\
\hline 4 & 0.1153 & 0.0886 & 0.1243 & 0.0990 & 0.1174 & 0.0936 & 0.1225 & 0.0974 \\
\hline 6 & 0.1266 & 0.0958 & 0.1519 & 0.1275 & 0.1294 & 0.1028 & 0.1508 & 0.1261 \\
\hline 8 & 0.1506 & 0.1274 & 0.1969 & 0.1657 & 0.1565 & 0.1345 & 0.1973 & 0.1638 \\
\hline 10 & 0.1916 & 0.175 & 0.279 & 0.208 & 0.2012 & 0.1818 & 0.2815 & 0.2072 \\
\hline 12 & 0.2668 & 0.2393 & 0.3858 & 0.2622 & 0.2811 & 0.2479 & 0.3934 & 0.2637 \\
\hline 14 & 0.3941 & 0.3548 & 0.5200 & 0.3465 & 0.4197 & 0.3594 & 0.5337 & 0.3517 \\
\hline 16 & 0.5815 & 0.4949 & 0.6931 & 0.5039 & 0.6231 & 0.5040 & 0.7174 & 0.5198 \\
\hline 18 & 0.7967 & 0.6480 & 0.9252 & 0.6226 & 0.8454 & 0.6712 & 0.9669 & 0.6448 \\
\hline 20 & 0.9331 & 0.8002 & 0.956 & 0.7619 & 0.9817 & 0.835 & 1 & 0.7936 \\
\hline
\end{tabular}

The PSNR performance of the received Watermarked image over the SC-FDMA systems for MMSE and ZF equalizer is studied. The PSNR values of the received Watermarked image for the IFDMA and the LFDMA systems at MMSE and ZF equalizer are tabulated in Table 2 for DCT and DWT watermarking technique, respectively. Figures 8 and 9 show the variations of the PSNR of the received watermarked image with the SNR for the SCFDMA system at different Equalizers, MMSE and ZF , and subcarriers mapping schemes, localized and distributed.

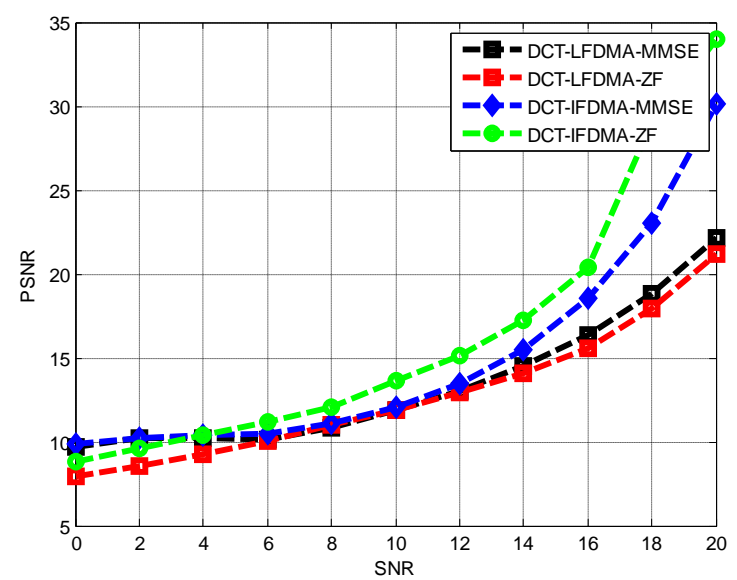

Fig. 8 PSNR values at different SNR of the received DCT watermarked image over the IFDMA and the LFDMA systems for MMSE and ZF equalizer.

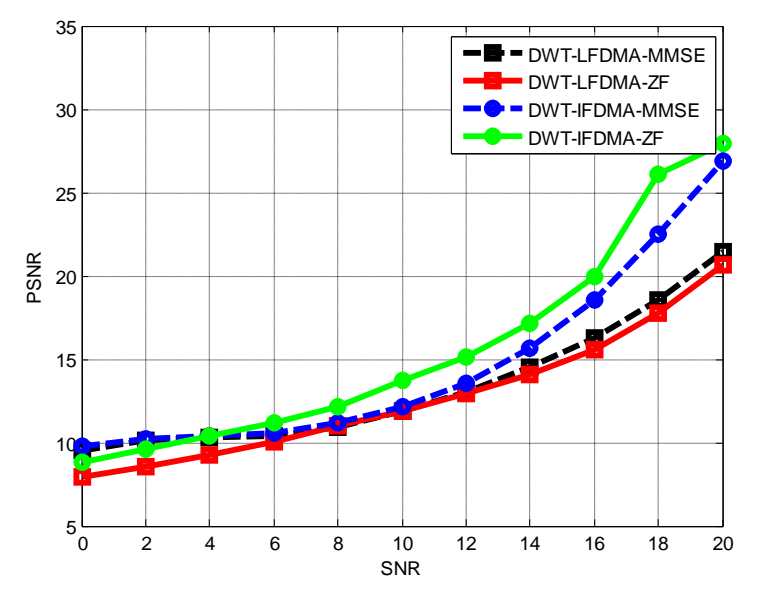

ig. 9 PSNR values at different SNR of the received DWT watermarked image over the IFDMA and the LFDMA systems for MMSE and ZF equalizer.

The correlation coefficient $(\mathbf{C r})$ between the original watermark and the extracted watermark for MMSE and ZF equalizer is studied for the SC-FDMA systems. The $\mathbf{C r}$ values of the extracted watermark for the IFDMA and the LFDMA systems at MMSE and ZF equalizer are tabulated in Table 3 for DCT and DWT watermarking technique, respectively. Figures 10 and 11 show the variations of the $\mathbf{C r}$ of the extracted watermark with the SNR for the SC-FDMA system at different Equalizers, MMSE and ZF , and subcarriers mapping schemes, localized and distributed. 


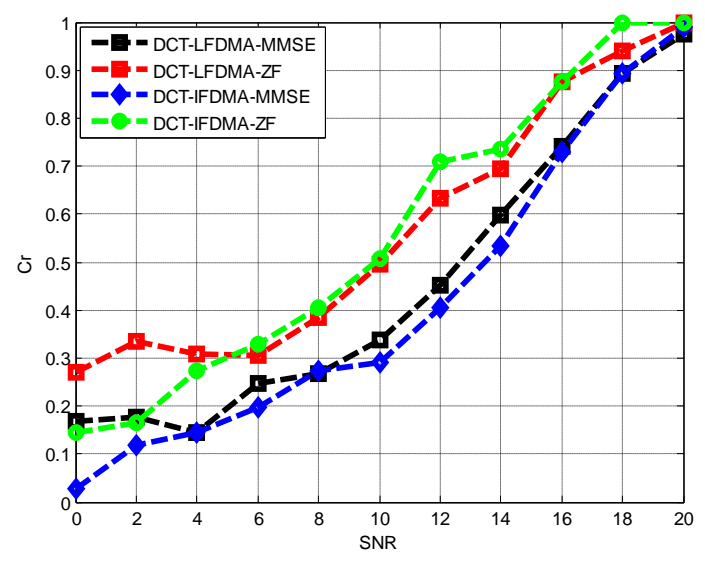

Fig. $10 \mathrm{Cr}$ vs. SNR of the extracted watermark based DCT over the IFDMA and the LFDMA systems for MMSE and ZF equalizer.

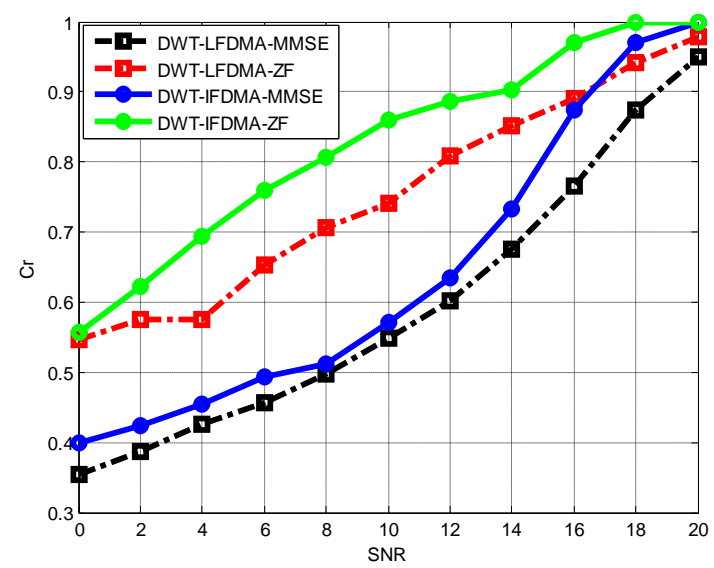

$11 \mathrm{Cr}$ vs. SNR of the extracted watermark based DWT over the IFDMA and the LFDMA systems for MMSE and ZF equalizer.

The SSIM performance of the received watermarked image over the SC-FDMA systems for MMSE and ZF equalizer is studied. The SSIM values of the received Watermarked image for the IFDMA and the LFDMA systems at MMSE and ZF equalizer are tabulated in Table 4 for DCT and DWT watermarking technique, respectively. Figures 12 and 13 show the variations of the SSIM of the received Watermarked image with the SNR for the SCFDMA system at different Equalizers, MMSE and ZF ,and subcarriers mapping schemes, localized and distributed.

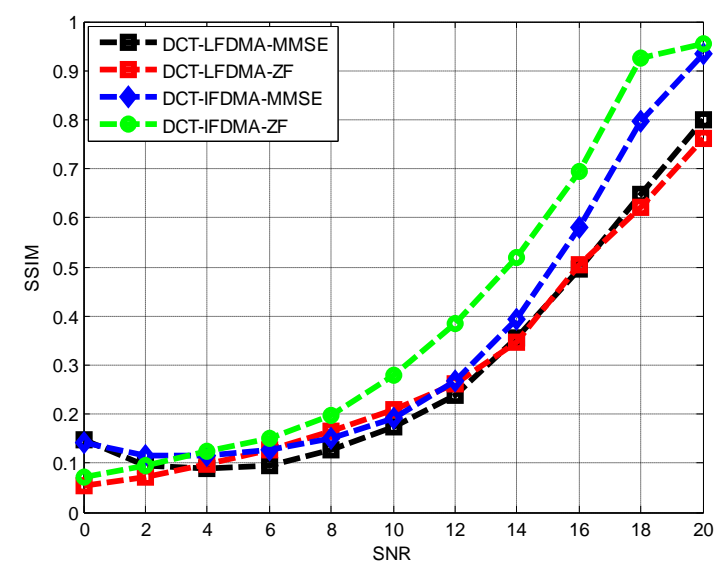

Fig. 12 SSIM vs. SNR of the received watermarked image based DCT over the IFDMA and the LFDMA systems for MMSE and ZF equalizer.

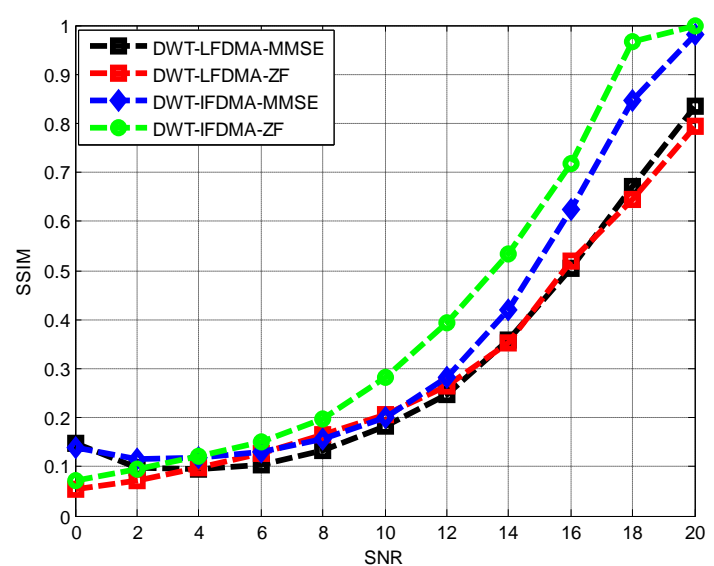

Fig.13 SSIM vs. SNR of the received watermarked image based DWT over the IFDMA and the LFDMA systems for MMSE and ZF equalizer.

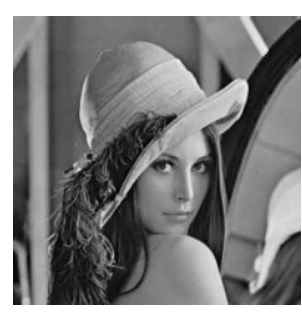

(a)

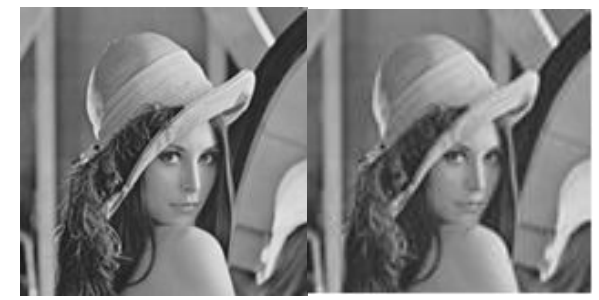

(c) $\mathrm{PSNR}=34.090$

(d) PSNR $=27.939$

Fig. 14: A comparison between the transmitted watermarking images after DCT and DWT techniques. (a) original image, (b)watermark image, (c) transmitted DCT watermarked image and (d) transmitted DWT watermarked image, respectively.

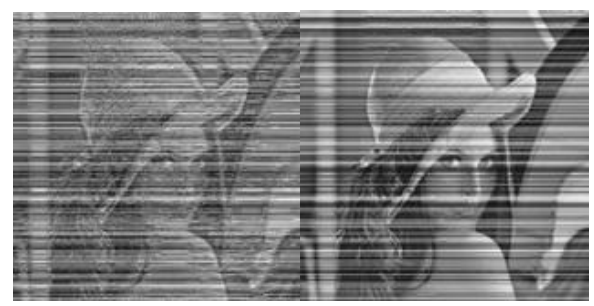

(a) IFDMA- MMSE

(b) IFDMA- ZF

PSNR $=10.421$

$\mathrm{PSNR}=10.766$

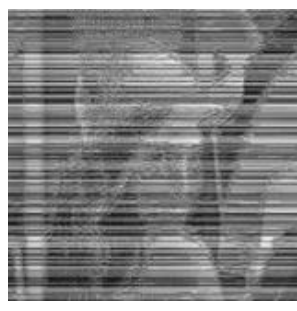

(c) LFDMA- MMSE PSNR $=10.163$

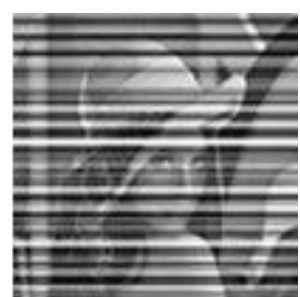

(d) LFDMA-ZF PSNR $=9.587$ 


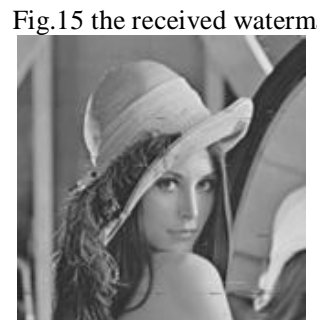

(a) IFDMA- MMSE PSNR $=30.149$

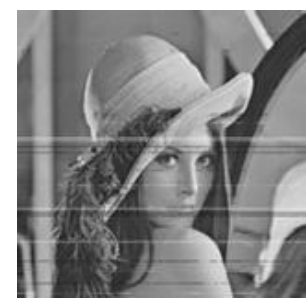

(c) LFDMA- MMSE PSNR $=22.105$

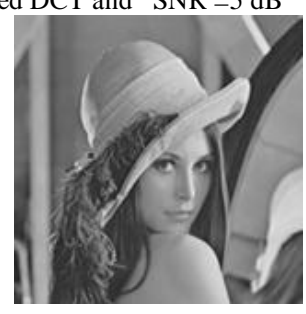

(b) IFDMA- ZF

PSNR $=34.020$

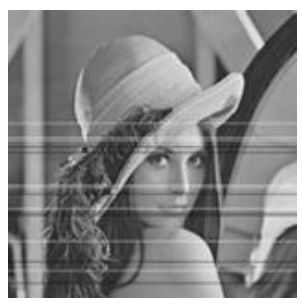

(d) LFDMA-ZF

$\mathrm{PSNR}=21.170$

Fig. 16 the received watermarked image based DCT and $S N R=20 \mathrm{~dB}$

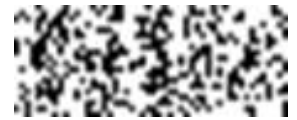

(a) IFDMA- MMSE

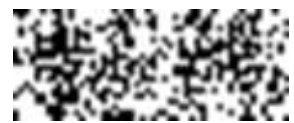

(c) LFDMA- MMSE

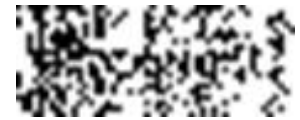

(b) IFDMA- ZF

Fig.17 the extracted watermark image based $\mathrm{DCT}$ and $\mathrm{SNR}=5 \mathrm{~dB}$

\section{Copyright}

(a) IFDMA- MMSE

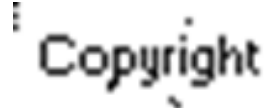

(c) LFDMA- MMSE

(d) LFDMA-ZF

Fig.18 the extracted watermark image based DCT and SNR $=20 \mathrm{~dB}$
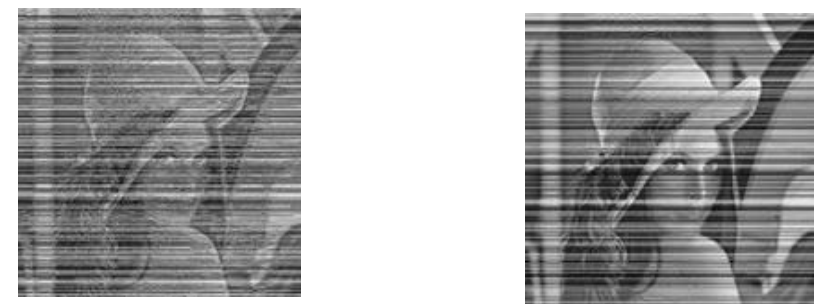

(a) IFDMA- MMSE

(b) IFDMA- ZF

PSNR $=10.4247$

PSNR $=10.8198$
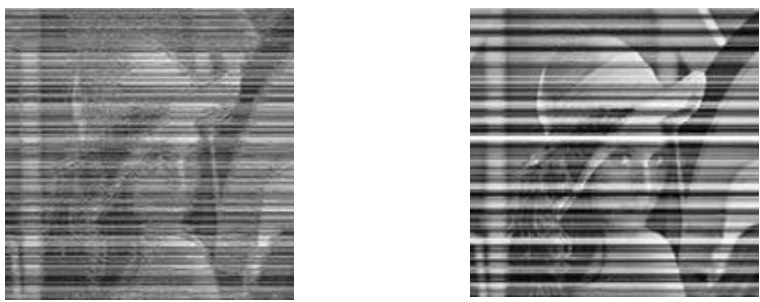
(c) LFDMA-MMSE
(d) LFDMA-ZF
$\mathrm{PSNR}=10.3141$
$\mathrm{PSNR}=9.597$

Fig.19 the received watermarked image based DWT and $\mathrm{SNR}=5 \mathrm{~dB}$
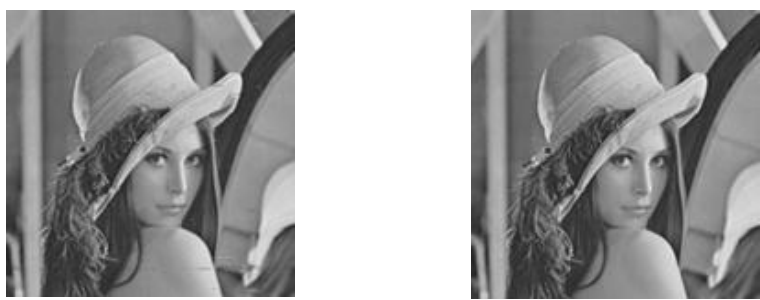

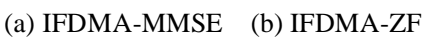

PSNR $=26.923 \quad$ PSNR $=27.939$
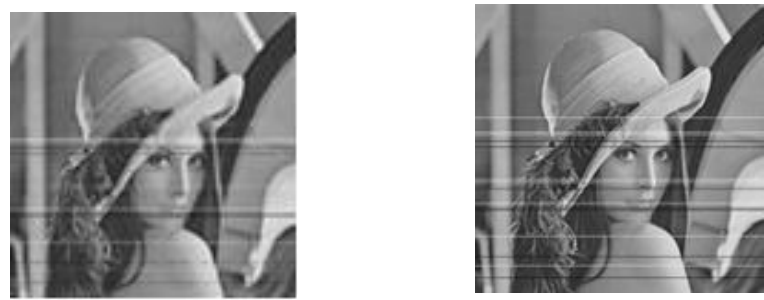
(c) LFDMA-MMSE
(d) LFDMA-ZF
PSNR $=21.446$
$\mathrm{PSNR}=20.678$

Fig.20 the received watermarked image based DWT and $S N R=20 \mathrm{~dB}$

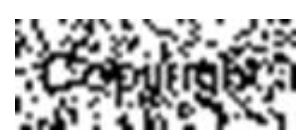

(a) IFDMA- MMSE

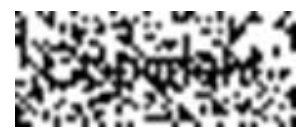

(c) LFDMA- MMSE LFDMA-ZF

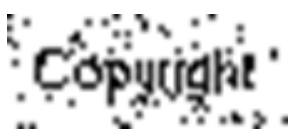

(b) IFDMA- ZF
Fig.21 the extracted watermark

$$
\text { based DWT and SNR }=5 \mathrm{~dB}
$$

\section{Copyright}




\section{Copyright}

(c) LFDMA- MMSE

\section{$\because$}

(d) LFDMA-ZF

\section{Copyright}

Fig. 22 the extracted watermark image based DWT and $\mathrm{SNR}=20 \mathrm{~dB}$

Lena image of size $512 \times 512$ is used as a cover image and copyright image of size $50 \times 20$ is used as a water mark image as shown in Fig. 14(a,b). Figure 14(c,d) show transmitted DCT watermarked and transmitted DWT watermarked, respectively.

Figures 15-22 show an example of received watermarked image and the extracted watermark image after transmission over SC-FDMA system. Figures 15-18 show the received watermarked image and the extracted watermark image for DCT watermarking technique at SNR $=5,20 \mathrm{~dB}$. Figures 19-22 show received watermarked image and the extracted watermark image for DWT watermarking technique at $\mathrm{SNR}=5,20 \mathrm{~dB}$.

It is clear that the IFDMA system gives better PSNR, $\mathrm{Cr}$ and SSIM values than the LFDMA system for both ZF and MMSE equalizers because the interleaved systems are more robust against the burst errors. Also, the IFDMA system performance with ZF equalizer is better that over the IFDMA system with MMSE equalizer.

For more investigation, the histogram performance metric is studied. The histograms of original, transmitted DCT watermarked and transmitted DWT watermarked images are displayed in Figure 23. Figures 24-27 display the histogram of the received watermarked image for DCT and DWT Watermarking techniques at $\mathrm{SNR}=5,20 \mathrm{~dB}$, respectively. The Comparison Between histograms of original images with transmitted and received watermarked histogram show that watermarked images maintain original image information.

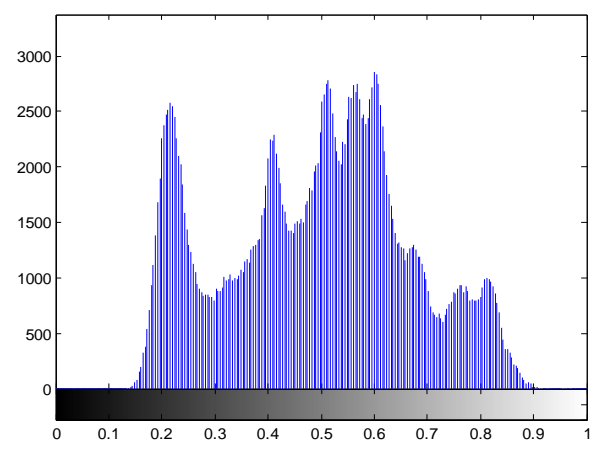

(a)

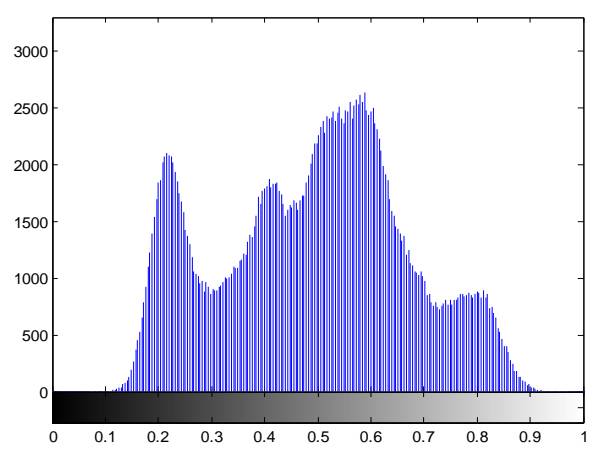

(b)

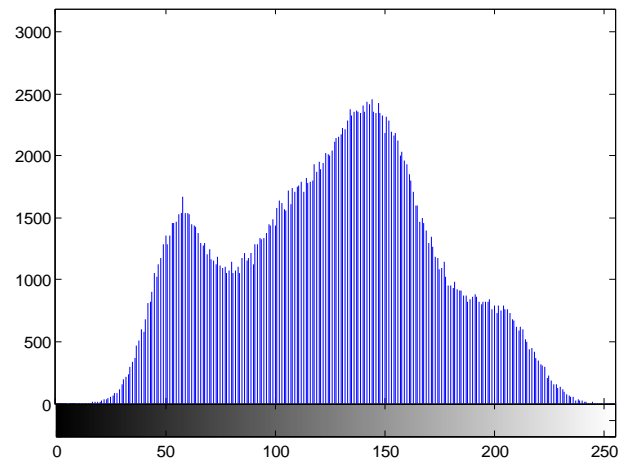

(c)

Fig. 23(a,b,c) Histogram of original image, transmitted DCT watermarked and transmitted DWT watermarked, respectively

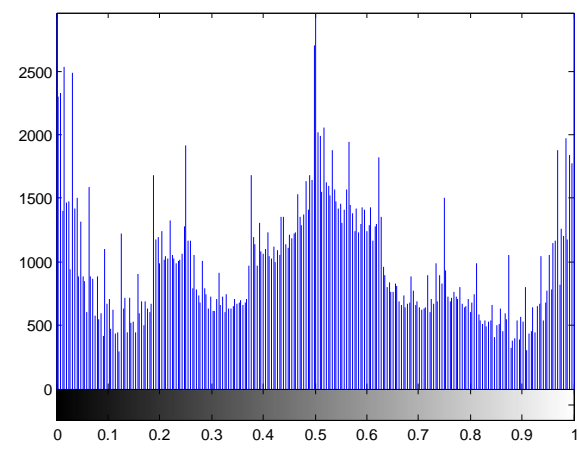

(a) IFDMA- MMSE

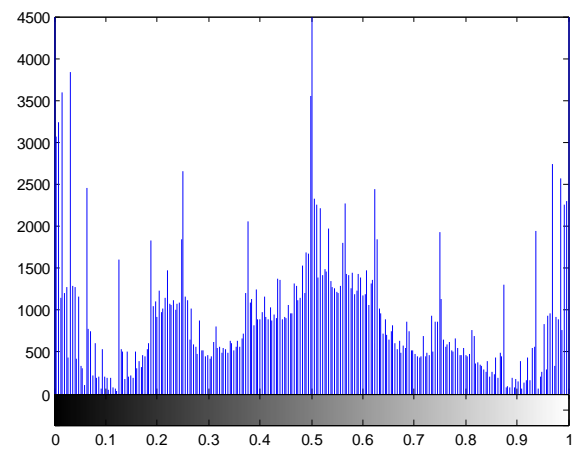

(b) IFDMA- ZF 


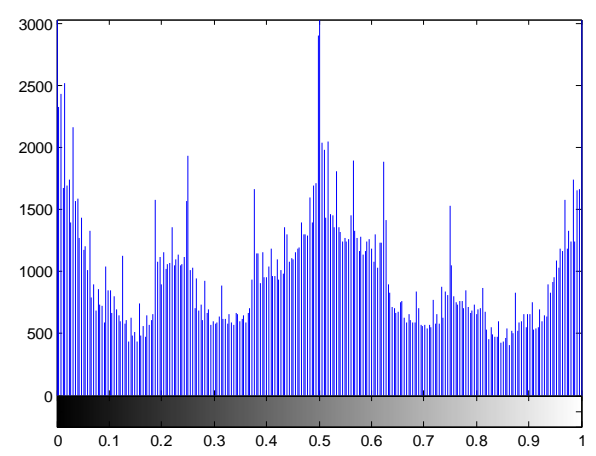

(c) LFDMA-MMSE

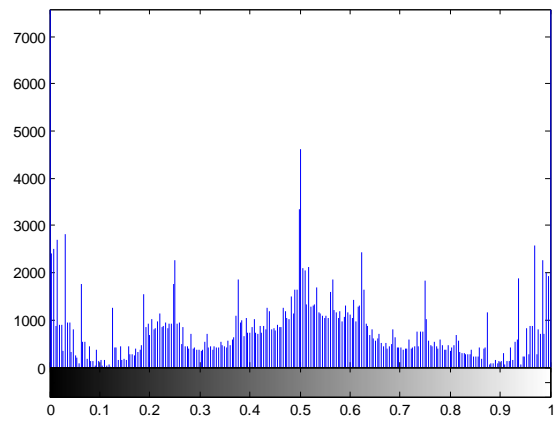

(d) LFDMA- ZF

Fig.24 Histogram of the received watermarked image based DCT, SNR $=5 \mathrm{~dB}$

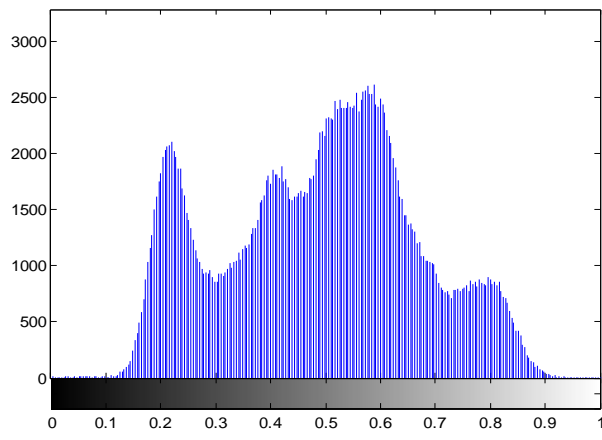

(a) IFDMA- MMSE

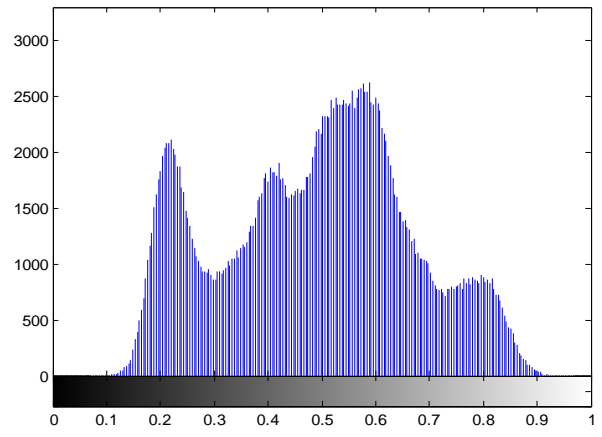

(b) IFDMA- ZF

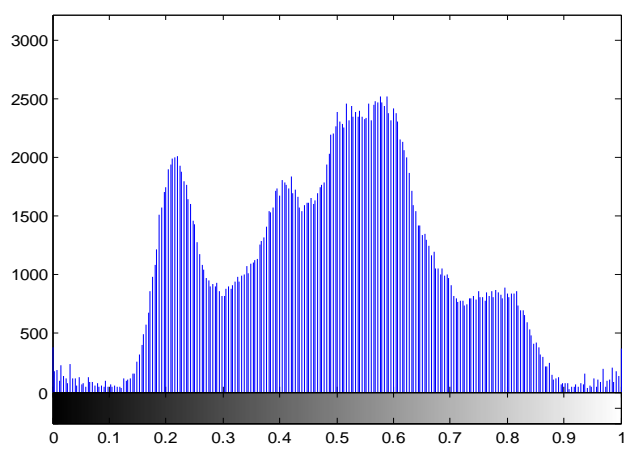

(c) LFDMA- MMSE

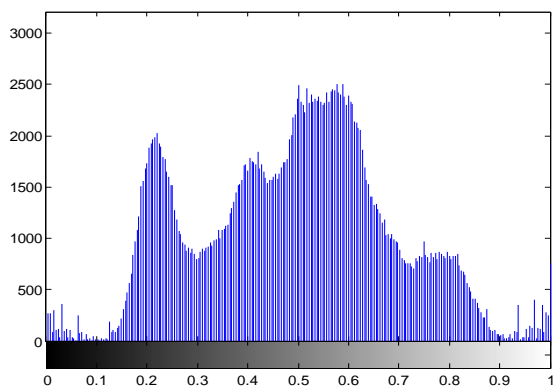

(d) LFDMA- ZF

Fig.25 Histogram of the received watermarked image based DCT, SNR $=20 \mathrm{~dB}$

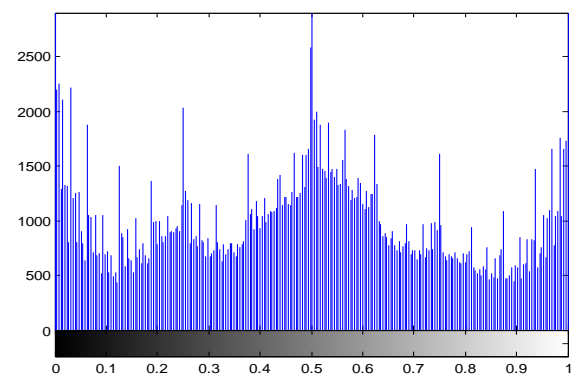

(a) IFDMA- MMSE

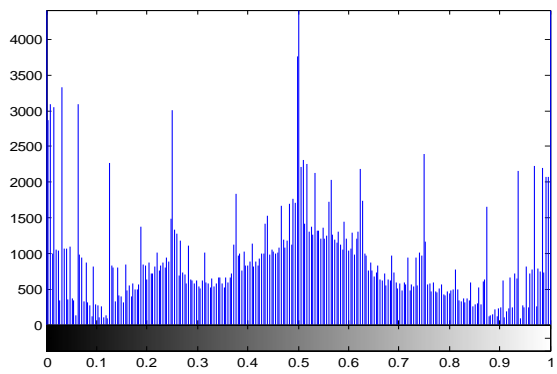

(b) IFDMA- ZF

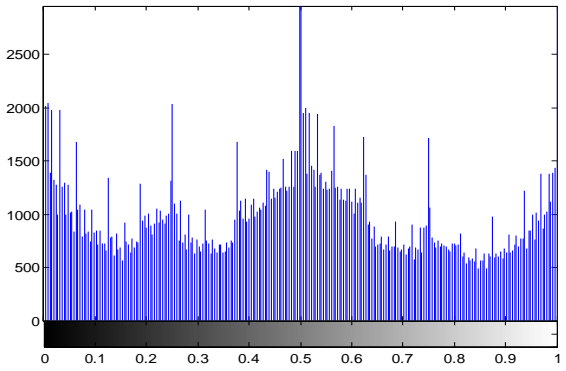

(c) LFDMA- MMSE 


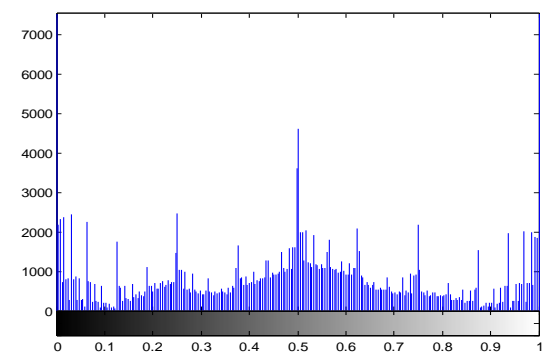

(d) LFDMA- ZF

Fig.26 Histogram of the received watermarked image based DWT,SNR $=5 \mathrm{~dB}$

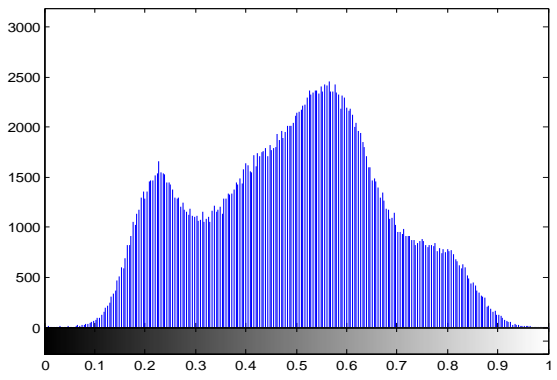

(a) IFDMA- MMSE

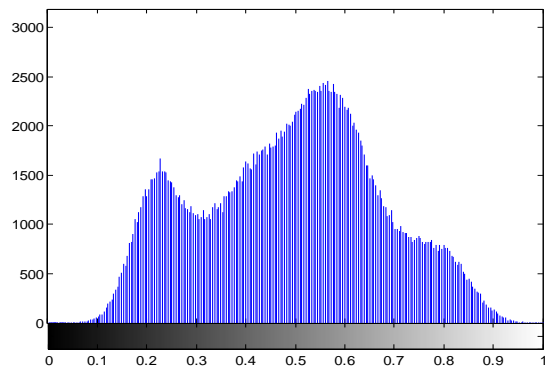

(b) IFDMA- ZF

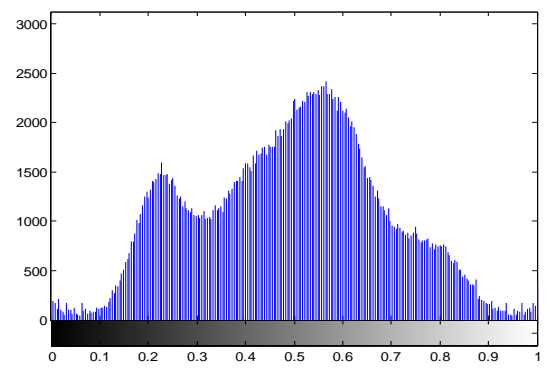

(c) LFDMA- MMSE

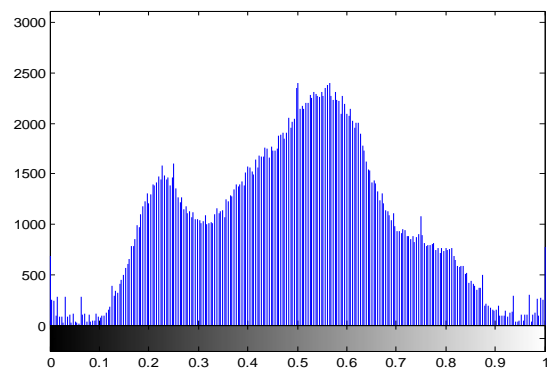

(d) LFDMA- ZF

Fig.27 Histogram of the received watermarked image based DWT,SNR $=20 \mathrm{~dB}$

\section{Conclusion}

In this paper, the image watermarking performances after transmission over SC-FDMA system have been studied for different types of equalizers. Simulations results show that the quality of the received images over the IFDMA system is better than that over the LFDMA system for both ZF and MMSE equalizers. Also, the simulation results show that the clarity of the received image over the IFDMA system with ZF equalizer is better than that over the IFDMA system with MMSE equalizer. This indicates that IFDMA system with ZF equalizer has better performance.

\section{References}

[1] Y. Shouyiand M. Xiaommi, "Performance Analysis of Digital Watermarking Through OFDM Channels," IEEE International Symposium on Microwave, Antenna, Propagation and EMC Technologies for Wireless Communications Proceedings, 2005.

[2] M. A. Abd El-Hamed, M. I. Dessouky, F. Shawki, M. K. Ibrahim, S.El-Rabaieand F. E. Abd El-Samie, "Wavelet-Based SC-FDMA System," 29th National Radio Science Conference(NRSC 2012)April $10-12,2012$, Faculty of Engineering/Cairo University, Egypt.

[3] A. Akter and M. AhsanUllah, "Digital Watermarking With A New Algorithm," IJRET: International Journal of Research in Engineering and Technology, eISSN: 2319-1163 |pISSN: 2321-7308, Volume: 03 Issue: 03 | Mar-2014.

[4] S. A. S. Abdelwahab, "Robust Watermarking Algorithms for Content Authentication and Secure ImageTransmission through Single Carrier Frequency DivisionMultiple Access,"Information Technology \& Electrical Engineering,Volume 3, Issue1, ISSN: - 2306-708X, February 2014.

[5] A. Elbehery, S. A. S. Abdelwahab, M. Abd EL Naby, E.S. Hassan, S. Elaraby, "Efficient Image Transmission Over the Single Carrier Frequency Division MultipleAccess System Using Chaotic Interleaving," 29th National RadioScienceConference(NRSC 2012), Faculty of Engineering/Cairo University, Egypt,April 10 -12, 2012.

[6] F. S. Al-Kamali, A. A. Qasem, S. A. Abuasbaa, G. A. Qasem, "SCFDMA and OFDMA: An Efficient WirelessImage Transmission Schemes," Journal of Control and Systems Engineering 2016, Vol. 4 Iss. 1, PP. 74-83

[7] F. E. Abd El-Samie, F. S. Al-kamali, A.Y. Al-nahari, M. I. Dessouky, "SC -FDMA for Mobile Communications," Taylor and Francis Group, LLC, 2010.

[8] P.Parashar and R. K. Singh, "A Survey: Digital Image Watermarking Techniques,"International Journal of Signal Processing, Image Processing and Pattern Recognition Vol. 7, No. 6 (2014)

[9] P. Dabas and K. Khanna, "A Study on Spatial and Transform DomainWatermarking Techniques," International Journal of Computer Applications (0975 - 8887), Volume 71- No.14, May 2013.

[10] A. K.Jain, "Fundamentals of Digital Image Processing, Englewood Cliffs, NJ, Prentice Hall”, 1989, pp. 150-153.

[11] M. A. M. El-Bendary, "FEC merged with double security approach based on encrypted image steganography for different purpose in the presence of noise and different attacks," Springer Science+BusinessMedia New York 2017.

[12] P. Ndajah, H. Kikuchi, M. Yukawa,H. Watanabe and S.Muramatsu, "SSIM Image Quality Metric ForDenoised Images," Advances In Visualization, Imaging and Simulation, ISSN: 1792-6130, September, 2010 .

[13] A. N. Patel and D. J. Shah, "SSIM based image quality assessment for vector quantization based lossy image compression using LZW coding," University Journal of Research Vol. 01, Issue 01 (2015). 